Brit. J. vener. Dis. (1958), 34, 38.

\title{
TRIAL OF SIGMAMYCIN IN NON-SPECIFIC URETHRITIS*
}

\author{
BY \\ J. K. OATES \\ Whitechapel Clinic, The London Hospital
}

Sigmamycin is the name given to a combination of two antibiotics, namely tetracycline hydrochloride and oleandomycin. It is reputed to show a synergistic action, as the inhibitory concentrations of this combination are lower than those of the individual components added together.

Sigmamycin was used in the treatment of two groups of patients suffering from non-specific urethritis (N.S.U.). The use of the term N.S.U. is synonymous with the "abacterial urethritis" of Harkness. All the patients complained of urethral discharge and slight local discomfort. Several had frequency of micturition but none severe dysuria. These patients were unselected and two dosage schedules were employed. The first group of patients numbered fifteen and each received $500 \mathrm{mg}$. Sigmamycin orally every $6 \mathrm{hrs}$ for 5 days. The results are shown in Table $\mathrm{I}$.

TABLE I

RESULTS IN GROUP 1

\begin{tabular}{c|c|c|c|c}
\hline $\begin{array}{c}\text { Follow-up Period } \\
\text { (days) }\end{array}$ & $\begin{array}{c}\text { No. } \\
\text { Followed }\end{array}$ & Failures & Relapses & Defaulters \\
\hline 0 & 15 & 0 & 0 & 0 \\
\hline $1-7$ & 15 & 0 & 0 & 1 \\
\hline $8-14$ & 14 & 0 & 0 & 0 \\
\hline $15-21$ & 14 & 0 & 1 & 1 \\
\hline $22-27$ & 12 & 0 & 1 & 0 \\
\hline $30-60$ & 11 & 0 & 1 & 1 \\
\hline $60-90$ & 9 & 0 & 0 & 0 \\
\hline
\end{tabular}

Nine patients had had previous attacks of N.S.U. within the preceding year and the two patients who relapsed after treatment were in this group. Of eight patients who completed tests of cure, only one was found to have chronic prostatitis. This was attributed to his attack of N.S.U. as he had no past history of genital disease. The only toxic effect noted was slight diarrhoea, which occurred in one case and lasted a week.

\footnotetext{
*Received for publication October 18, 1957.
}

In the second group of patients, eighteen in number, each received $250 \mathrm{mg}$. Sigmamycin orally every 6 hrs for 5 days. The results are shown in Table II.

TABLE II

RESULTS IN GROUP 2

\begin{tabular}{c|c|c|c|c}
\hline $\begin{array}{c}\text { Follow-up Period } \\
\text { (days) }\end{array}$ & $\begin{array}{c}\text { No. } \\
\text { Followed }\end{array}$ & Failures & Relapses & Defaulters \\
\hline 0 & 0 & 0 & 0 & 0 \\
\hline $1-7$ & 18 & 1 & 0 & 2 \\
\hline $8-14$ & 15 & 1 & 0 & 1 \\
\hline $15-21$ & 13 & 0 & 0 & 1 \\
\hline $22-27$ & 12 & 0 & 0 & 0 \\
\hline $30-60$ & 12 & 1 & 2 & 0 \\
\hline $60-90$ & 9 & 0 & 0 & 0 \\
\hline
\end{tabular}

Ten patients in this series had suffered attacks of N.S.U. within the preceding year and one of these relapsed after treatment. The other patient who relapsed had no previous history of genital disease. Two failures of treatment occurred, but in one of these cases Trichomonas vaginalis was found and the urethritis persisted for many weeks. The second case was complicated by two severe attacks of conjunctivitis associated with arthralgia, and was considered to be a forme fruste of Reiter's syndrome.

Ten patients completed all tests of cure, but three were found to have chronic prostatitis. Two of these patients were known to have had chronic prostatitis in the past, and it is doubtful whether that condition had previously been cured. No toxic effects were encountered. No stricture was found in either group.

\section{Discussion}

The assessment of the value and relative efficacy of any drug in the treatment of N.S.U. is fraught with many difficulties, some common to any therapeutic trial, others peculiar to this infection. Cases 
of N.S.U. vary greatly in their clinical severity and it is therefore very difficult to obtain strictly comparable groups. The cessation of urethral discharge may be misleading as examination after holding the urine overnight may reveal the presence of a profuse discharge. In addition, relapse or reinfection is extremely common, and therefore longterm observation is necessary, a state of affairs rarely possible to achieve in a venereal disease clinic. Furthermore, there is little knowledge of the natural history of the disease and the effects of therapy cannot be compared with the results to be expected from withholding all treatment. Bearing these facts in mind, any conclusions as to the relative efficiency of any drug must be tentative, but as far as the evidence goes it suggests that Sigmamycin is a very effective method of treating N.S.U. and is at least equal in effect to those drugs generally accepted as being of the greatest value in the treatment of this infection.

\section{Summary}

Sigmamycin in dosage of 500 or $250 \mathrm{mg}$. 6-hrly, for 5 days appears to be a very effective method of treating N.S.U. With the lower dosage there were no toxic effects and complications of the disease such as asymptomatic prostatitis occurred in few instances.

It appears that the chance of cure is better when there is no previous history of urethritis.

The only drawback to the use of the drug in routine practice is its present high cost. 